\title{
Pembrolizumab combined with lenvatinib as non-first-line therapy in patients with refractory biliary tract carcinoma
}

\author{
Jianzhen Lin ${ }^{1 \#}$, Xu Yang ${ }^{1 \#}$, Junyu Long ${ }^{1 \#}$, Songhui Zhao ${ }^{2 \#}$, Jinzhu Mao ${ }^{1}$, Dongxu Wang ${ }^{1}$, Yi Bai ${ }^{1}$ Jin Bian $^{1}$, \\ Lei Zhang ${ }^{1}$, Xiaobo Yang ${ }^{1}$, Anqiang Wang ${ }^{3}$, Fucun Xie ${ }^{1}$, Weiwei Shi ${ }^{2}$, Huayu Yang ${ }^{1}, J_{i e ~}$ Pan $^{4}, \mathrm{Ke} \mathrm{Hu}^{5}$, \\ Mei Guan ${ }^{6}$, Lin Zhao $^{6}$, Li Huo ${ }^{7}$, Yilei Mao ${ }^{1}$, Xinting Sang ${ }^{1}$, Kai Wang ${ }^{2}$, Haitao Zhao ${ }^{1}$ \\ ${ }^{1}$ Department of Liver Surgery, Peking Union Medical College Hospital, Chinese Academy of Medical Sciences \& Peking Union Medical College \\ (CAMS \& PUMC), Beijing 100730, China; ${ }^{2}$ OrigiMed, Shanghai 200135, China; ${ }^{3}$ Department of Gastrointestinal Surgery, Peking University Cancer \\ Hospital \& Institute, Beijing 100142, China; ${ }^{4}$ Department of Radiology, ${ }^{5}$ Center of Radiotherapy, ${ }^{6}$ Department of Medical Oncology, ${ }^{7}$ Department \\ of Nuclear Medicine, Peking Union Medical College Hospital, Beijing 100730, China \\ Contributions: (I) Conception and design: J Lin, X Sang, H Zhao; (II) Administrative support: W Shi, H Yang, Y Mao, X Sang, K Wang, H Zhao; \\ (III) Provision of study material or patients: J Pan, K Hu, M Guan, L Zhao, L Huo, Y Mao, X Sang, K Wang, H Zhao; (IV) Collection and \\ assembly of data: J Lin, X Yang, J Long, J Mao, D Wang, Y Bai, J Bian, L Zhang, X Yang, A Wang, F Xie, J Pan, K Hu, M Guan, L Zhao, L Huo; \\ (V) Data analysis and interpretation: J Lin, X Yang, J Long, S Zhao, K Wang, H Zhao; (VI) Manuscript writing: All authors; (VII) Final approval of \\ manuscript: All authors. \\ \#These authors contributed equally to this work. \\ Correspondence to: Haitao Zhao, MD. Department of Liver Surgery, Peking Union Medical College Hospital, Chinese Academy of Medical Sciences \\ and Peking Union Medical College (CAMS \& PUMC), \#1 Shuaifuyuan, Wangfujing, Beijing 100730, China. Email: zhaoht@pumch.cn; Kai Wang, \\ MD, PhD. OrigiMed, Shanghai 200135, China. Email: wangk@origimed.com.
}

Background: A therapeutic strategy involving combined treatment with lenvatinib plus pembrolizumab (LEP) has demonstrated a relatively high antitumor response in several solid tumors; however, the efficacy and safety of LEP in patients with refractory bile tract carcinoma (BTC) remains unknown.

Methods: This is a single-arm study for a preliminary assessment of the efficacy and tolerability of LEP in patients who experienced progression from prior systemic treatments. Pre-treatment tumor tissues were collected to retrospectively evaluate the expression status of PDL1.

Results: Thirty-two patients received second-line and above treatment with LEP. Overall, the objective response rate (ORR) was $25 \%$, the disease control rate (DCR) was $78.1 \%$, and the clinical benefit rate (CBR) was $40.5 \%$. The median progression-free survival (PFS) was 4.9 months (95\% CI: 4.7-5.2 months), and the median overall survival (OS) was 11.0 months (95\% CI: 9.6-12.3 months). For tolerability, no grade 5 serious adverse events (AEs) were reported. All patients had any-grade AEs, and $59.3 \%$ of the patients experienced grade 3 AEs, while only 1 patient experienced a grade $4 \mathrm{AE}$ of stomach bleeding. Fatigue was the most common AE, followed by hypertension and elevated aminotransferase levels. Retrospective analysis for PDL1 expression revealed that PDL1 positive tumor cells were associated with improved clinical benefits and survival outcomes.

Conclusions: LEP is a promising alternative as a non-first-line therapeutic regimen for patients with refractory BTC. Furthermore, well-designed prospective clinical trials with a control arm are still needed to obtain more evidences to confirm the efficacy and safety of this particular regimen as well as the role of PDL1 expression.

Keywords: Pembrolizumab; lenvatinib; bile tract cancer; PD1; PDL1

Submitted Mar 02, 2020. Accepted for publication Apr 26, 2020.

doi: $10.21037 /$ hbsn-20-338

View this article at: http://dx.doi.org/10.21037/hbsn-20-338 


\section{Introduction}

Biliary tract carcinoma (BTC) is a collective term encompassing intrahepatic cholangiocarcinoma (ICC), extrahepatic cholangiocarcinoma (ECC) and gallbladder cancer (GBC). The mortality of this type of cancer has increased over the past 25 years $(1,2)$, and its incidence is reported to be steadily rising $(3,4)$. As one of the most malignant tumors, survival continues to be poor in patients with BTC, with a median overall survival (OS) time of less than one years at advanced or metastatic stage, and the 5 -year OS rate of $5-15 \%(5,6)$.

Cisplatin plus gemcitabine is widely used as the firstline treatment in patients with unresectable BTC (7). However, for patients with metastatic BTC who experience progression even with chemotherapy, best supportive care (BSC) without antitumor intervention is routinely recommended since there is a lack of efficacious protocols as second-line and above treatment. The shortage of available antitumor regimens for patients with refractory BTC has plagued the oncologists for a long time. Largely based on mutation-guided precision oncology (8) and immunotherapy targeted PD1/PDL1, which have a wide antitumor spectrum and have been applied in other solid tumors (9), antitumor drugs such as pembrolizumab and antiangiogenic targeting agents are also used because there is no standard therapy for patients with chemotherapyresistant BTC (10-12).

In this report, we focused on the non-first-line combined therapy with lenvatinib plus pembrolizumab (LEP) in patients with refractory BTC. LEP has been granted as a "breakthrough designation" in hepatocellular carcinoma, endometrial carcinoma and renal cell cancer by the FDA (13). Previous prospective clinical trials have shown that more than one third of patients with advanced or metastatic types of these three cancers can obtain an objective response with tolerable adverse effects. These studies have motivated increasing clinical investigations of this regimen in patients with refractory cancers. Importantly, either lenvatinib or pembrolizumab has exhibited a satisfactory safety index in treating patients with unresectable BTC (14-16). Thus, we believe that LEP may become an encouraging alternative in patients with metastatic BTC who have experienced progression even after systemic chemotherapy.

Herein, we preliminarily estimate the therapeutic efficacy and safety of LEP as a non-first-line treatment in patients with advanced BTC. Out study also performed an exploratory analysis of histological PDL1 expression to correlate with therapeutic outcomes.
We present the following article in accordance with the CONSORT reporting checklist (available at http://dx.doi. org/10.21037/hbsn-20-338).

\section{Methods}

\section{Study design and patients}

This was a single-arm, investigator-initiated study to preliminarily assess the efficacy and tolerability of LEP as a non-first-line treatment in patients with advanced BTC who experienced disease progression from previous systemic chemotherapy. The demographic, surgical, pathological, regional and systemic treatment information were compiled and recorded. The protocol of this study was compliant with the principles of the Declaration of Helsinki and was also approved by the Institutional Review Board (IRB) and Ethics Committee (EC) of Peking Union Medical College Hospital (PUMCH). The study has registered at ClinicalTrials.gov (identifiers: NCT03895970).

The primary eligibility criteria included histologically confirmed BTC, including ICC, ECC or GBC, at least a previous systemic anti-BTC therapy, at least one measurable tumor lesion according to the RECIST-v1.1 criteria (17), ECOG performance status of $0-2$ and need for further systemic treatment for refractory or metastatic disease. These participants were required to be older than 18 years. We excluded patients for whom there were no pre-LEP antitumor treatments and those with mixed pathological tumor types. All participants were asked to provide written informed consent.

\section{Treatment and dosing}

Information regarding the dates of initiation and completion of treatment, initial dose, dose modifications, radiological evaluation, reason for therapeutic interruption and discontinuation, laboratory data and adverse events (AEs) during treatment were systematically collected. The prescription dosage of lenvatinib was $12 \mathrm{mg}$ (for patients with a body weight $\geq 60 \mathrm{~kg}$ ) or $8 \mathrm{mg}$ (for patients with a body weight $<60 \mathrm{~kg}$ ) orally once a day. For pembrolizumab, investigator-chosen doses included a fixed dosage of $200 \mathrm{mg}$ every 3 weeks ( $\mathrm{n}=11)$ or a fixed dosage of $3 \mathrm{mg} / \mathrm{kg}$ body weight every 3 weeks $(n=21)$.

\section{Assessments}

The objective response was measured according to the 
RECIST version 1.1 criteria (17) and was evaluated by professional radiologists at the center (PUMCH) who were blinded to the therapeutic outcomes and clinicopathological features. The rate of tumor growth during the treatment was quantified only for target lesions. To assess the rate of tumor growth and therapeutic response, computed tomography/magnetic resonance imaging (CT/MRI) or positron emission tomography (PET) CT/MRI images were regularly evaluated every two or three months, which required the essential records at baseline (before the initial dose of LEP), periodical evaluations during LEP treatment and a final evaluation of confirmed disease progression.

To assess therapeutic efficacy, the clinical outcomes included the objective response rate (ORR), disease control rate (DCR), progression-free survival (PFS), OS and clinical benefit rate (CBR). The ORR was determined as the proportion of patients who achieved a radiologically confirmed objective response including a complete response (CR) or partial response (PR). The DCR indicates the proportion of patients who experienced at least one radiologically confirmed objective response or stable disease (SD) during treatment with LEP. PFS refers to the time from the initial dose to the first radiologically confirmed progressive disease (PD) or death, and OS was defined as the time range from the date of the initial dose to the date of death. Patients who discontinued treatment due to non-PD events were censored at the date of the final dose. Patients who were still alive or had not experienced progression events at the date of data cut-off (January 1,2020) were censored. The CBR was defined as the proportion of patients who achieved a radiologically confirmed objective response (CR or PR) or who had PFS time longer than 6 months.

Tolerability and toxicity were reported by patients or clinicians, and were collected by the investigators, using the Common Terminology Criteria for Adverse Events (version $4.0)$ as a reference. The treatment-related toxicities from the date of the initial dose to 30 days after the final dose were recorded.

\section{Evaluation of PDL1 expression}

Whole sections from available formalin-fixed, paraffinembedded (FFPE) tumor specimens were used to perform immunohistochemistry (IHC). For each tissue slice, serial 4- to 5- $\mu \mathrm{m}$-thick sections were selected and placed on glass slides. The primary antibody used was anti-PDL1
(IHC 22C3 pharmDx, Dako North America, Agilent Technologies); the secondary antibodies were added to all sections, including the negative control slides. The evaluations of PDL1 expression were performed by independent pathologists, who were blinded to the clinicopathologic data, including the therapeutic response and survival time. PDL1 positivity or overexpression was defined as more than $5 \%$ positive expression in tumor cells.

\section{Statistical analysis}

The data from the cut-off date (January 1, 2020) of the analysis in this report were used to generate summaries of the baseline characteristics, therapeutic efficacies and AEs. The molecular marker analyses included treated patients with available data as of January 1, 2020. The PFS, OS, 6-month PFS and 12-month OS were all estimated by the Kaplan-Meier method, and the comparisons were analyzed using the log-rank test. Hazard ratios of each clinicopathological feature for PFS and OS were estimated by Cox proportional hazard modeling. To compare the individual variables, the $t$-test, Mann-Whitney $\mathrm{U}$ test, $\chi^{2}$ test and Fisher's exact test were performed as appropriate. A two-tailed $\mathrm{P}$ value $<0.05$ was considered significant. Statistical analyses were performed using SPSS (version 23) and R-3.5.1 software.

\section{Results}

\section{Patient characteristics}

A total of 32 patients with advanced BTC who received a non-first-line treatment of LEP. At the time of the initial treatment with LEP, the median age was 56.5 (IQR, 49.5-68.5) years, and 28 (87.5\%) patients had an ECOG performance status of $0-1$. For anatomical subtypes of BTC, 16 patients had ICC, 10 patients had ECC and 6 patients had GBC. All histopathological subtypes were adenocarcinomas. In total, 18 (56.25\%) patients had poorly differentiated BTC.

Regarding the risk factors for BTC in our cohort, 6 (19\%) patients had hepatitis B virus infections. All patients with chronic hepatitis received regular antiviral therapy during LEP treatment. In addition, 10 (31\%) patients had biliary stone and $11(34 \%)$ patients had a smoking habit, but no patients had a liver fluke infection (Table 1). 
Table 1 Patient baseline clinicopathological characteristics

\begin{tabular}{|c|c|}
\hline Parameters & Total $(\mathrm{N}=32)$ \\
\hline Age, years (median, IQR) & $56.5(49.5-68.5)$ \\
\hline \multicolumn{2}{|l|}{ Gender, n [\%] } \\
\hline Female & $14[44]$ \\
\hline Male & $18[56]$ \\
\hline \multicolumn{2}{|l|}{ ECOG performance, $\mathrm{n}$ [\%] } \\
\hline 0 & $8[25]$ \\
\hline 1 & 20 [62] \\
\hline 2 & $4[13]$ \\
\hline \multicolumn{2}{|l|}{ Tumor subtype, n [\%] } \\
\hline Intrahepatic cholangiocarcinoma & $16[50]$ \\
\hline Extrahepatic cholangiocarcinoma & $10[31]$ \\
\hline Gallbladder cancer & $6[19]$ \\
\hline \multicolumn{2}{|l|}{ Histology, n [\%] } \\
\hline Adenocarcinoma & $32[100]$ \\
\hline \multicolumn{2}{|l|}{ Differentiated histology, $\mathrm{n}$ [\%] } \\
\hline Poor & $18[56]$ \\
\hline Moderate & 9 [28] \\
\hline Unsure & $5[16]$ \\
\hline Hepatitis (HBV) infection, n [\%] & $6[19]$ \\
\hline Liver fluke infection, n (\%) & 0 \\
\hline Bile stone disease, $\mathrm{n}[\%]$ & $10[31]$ \\
\hline Habit of smoking, $\mathrm{n}$ [\%] & $11[34]$ \\
\hline \multicolumn{2}{|l|}{ Site of metastases, $\mathrm{n}(\%)$} \\
\hline Intrahepatic & $28(87.5)$ \\
\hline Lymph nodes & $28(87.5)$ \\
\hline Lung & $7(22.0)$ \\
\hline Bone & $3(9.0)$ \\
\hline \multicolumn{2}{|l|}{ Previous antitumor therapy, $\mathrm{n}$ [\%] } \\
\hline Radical surgery resection & $17[53]$ \\
\hline Systemic chemotherapy & 26 [81] \\
\hline Targeted therapy & $6[19]$ \\
\hline Regional radiotherapy or ablation & $9[28]$ \\
\hline Transarterial chemoembolization & $11[34]$ \\
\hline
\end{tabular}

ECOG, Eastern Cooperative Oncology Group; HBV, hepatitis type $B$ virus.

\section{Overview of disease stage and previous treatment}

At baseline before receiving LEP treatment, most patients had metastatic cancer in the liver $(28 / 32,87.5 \%)$, lymph nodes $(28 / 32,87.5 \%)$, lungs $(7 / 32,22 \%)$ and bone $(3 / 32$, $9 \%)$. Regarding prior treatments, 17 (53\%) patients underwent radical surgical resection. The therapeutic regimen of LEP was generally administered as a nonfirst-line treatment to patients with refractory disease, and all patients had previously received at least one line antitumor treatment. Regarding the regimens used in the previous treatment, $26(81 \%)$ patients experienced disease progression after gemcitabine-based or platinumbased chemotherapy. In total, 6 (19\%) patients received a single treatment with a tyrosine kinase inhibitor, including cabozantinib (2 cases), afatinib (2 cases), regorafenib (1 case) and everolimus (1 case). Palliative regional treatment with transcatheter arterial chemoembolization was performed alone in 11 patients, and with ablation or radiotherapy in 9 patients (Table 1).

\section{Treatment and efficacy}

For all participants in our cohort, the median duration of follow-up was 9.5 (IQR, 5.6-15.5) months, and the median duration time of treatment was 5.5 (IQR, 4.0-11.7) months. At the time of the analysis, 29 patients discontinued the treatment due to radiologically confirmed PD $(n=21)$ or intolerable AEs $(\mathrm{n}=8)$.

In the present cohort, all patients had complete radiological evaluations, and they were available for assessments of PFS and OS. Overall, 22 of the 32 (68.8\%) patients exhibited a decrease in tumor size from baseline (Figure 1A). Among these patients, 8 patients achieved an objective response, all of which were PRs, and no CR was observed. The median time to achieve an objective response was 3.0 (range, 2.0-6.5) months. In the population with available radiological assessments, the best response in 17 patients was SD. Therefore, the overall radiologicalconfirmed ORR was $25 \%$ (95\% CI: 9.1-40.9\%), and the DCR was $78.1 \%$ (95\% CI: 63-93.3\%, Table 2).

The survival outcomes of the enrolled patients were investigated in the entire cohort. Twelve patients are still alive and only 3 patients were lost to follow-up after LEP treatment due to disease progression. For the entire cohort, the median PFS was 4.9 (95\% CI: 4.7-5.2) months (Figure 1B), and the 

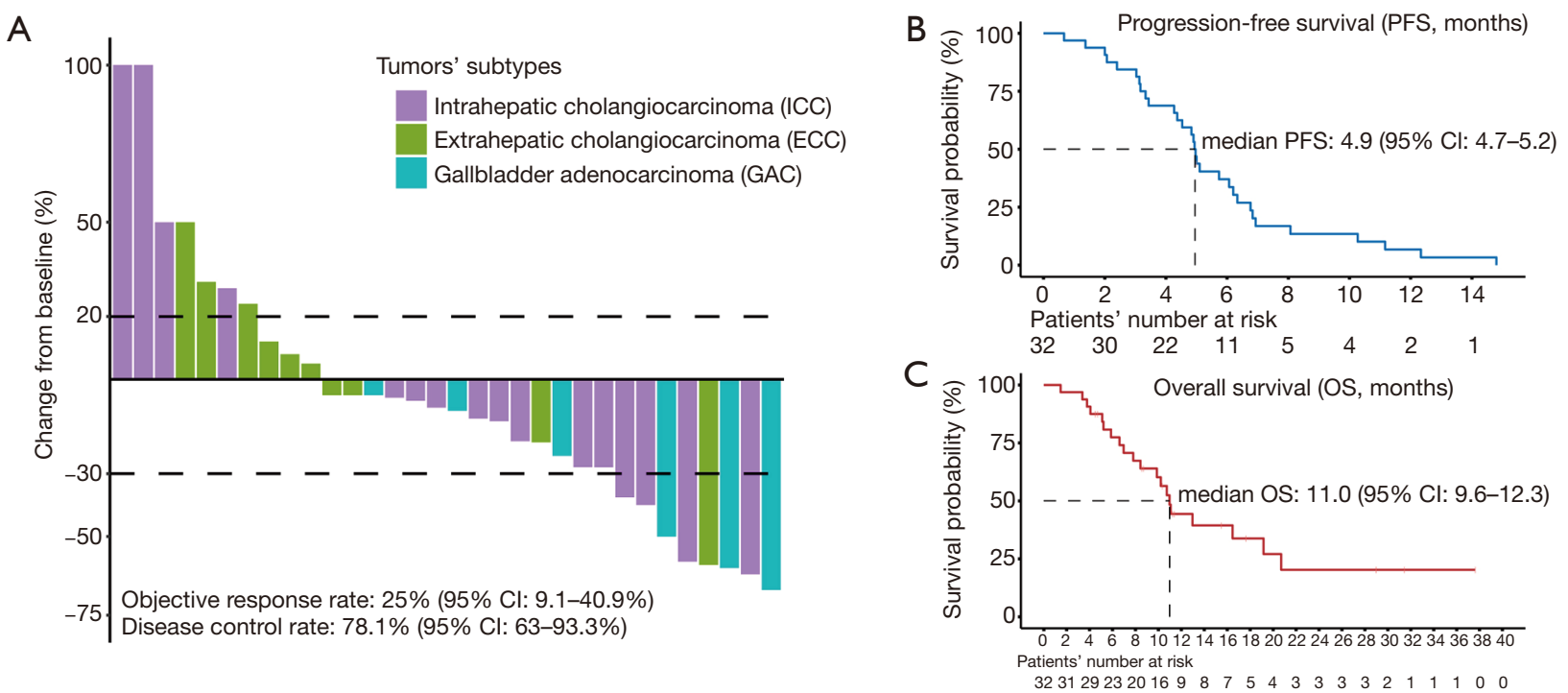

Figure 1 Therapeutic efficacy of lenvatinib combined with pembrolizumab (LEP) in patients with advanced biliary tract carcinoma. (A) Maximum percentage change in the sum of the diameters of the target lesions from baseline. The three different anatomical subtypes of biliary tract cancers are highlighted; (B) Kaplan-Meier estimation of progression-free survival of the entire cohort; (C) Kaplan-Meier estimation of overall survival of the entire cohort.

Table 2 Therapeutic efficacy of response and survival outcomes for 32 BTC patients treated by lenvatinib plus pembrolizumab

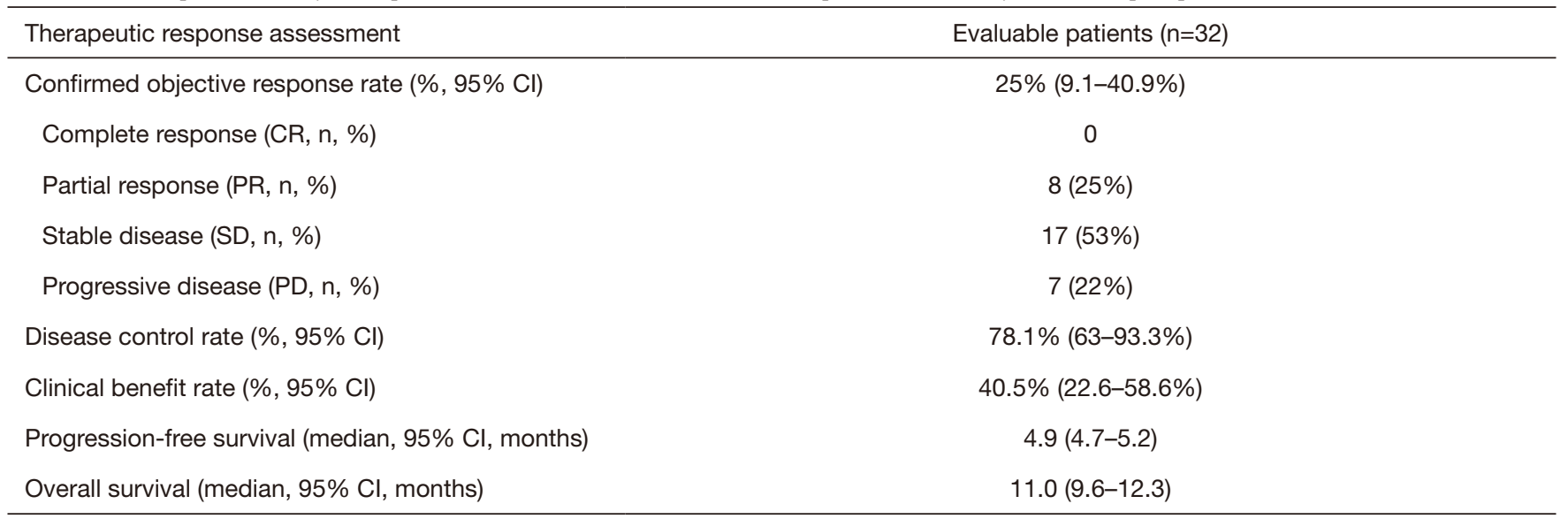

6-month PFS rate was $33.7 \%$ (95\% CI: $17-50.4 \%$ ). The median OS was 11.0 (95\% CI: 9.6-12.3) months (Figure 1C), and the 1-year OS rate was $39.4 \%$ (95\% CI: 20.6-58.2\%). We further determined the CBR in all assessment-available patients. Impressively, the CBR in all 32 patients was $40.5 \%$ (95\% CI: $22.6-58.6 \%)$.

\section{Tolerability and safety}

In total, for 32 patients with available safety assessments,
$12(37.5 \%)$ patients had a history of a dosage reduction in lenvatinib alone. Twenty-one (65.6\%) patients had history of at least one therapeutic interruption. All the recorded any-grade AEs inducing treatment termination were reversible, and there were no AEs-related deaths.

AEs during LEP treatment were reported in all 32 (100\%) patients, and no grade 5 AEs occurred. For severe AEs (SAEs), 19 (59.3\%) patients had grade 3 AEs, and only $1(3.1 \%)$ patient had a grade $4 \mathrm{AE}$ of stomach bleeding (Table 3). The most common AEs (any grade) were fatigue 
Table 3 Safety summary

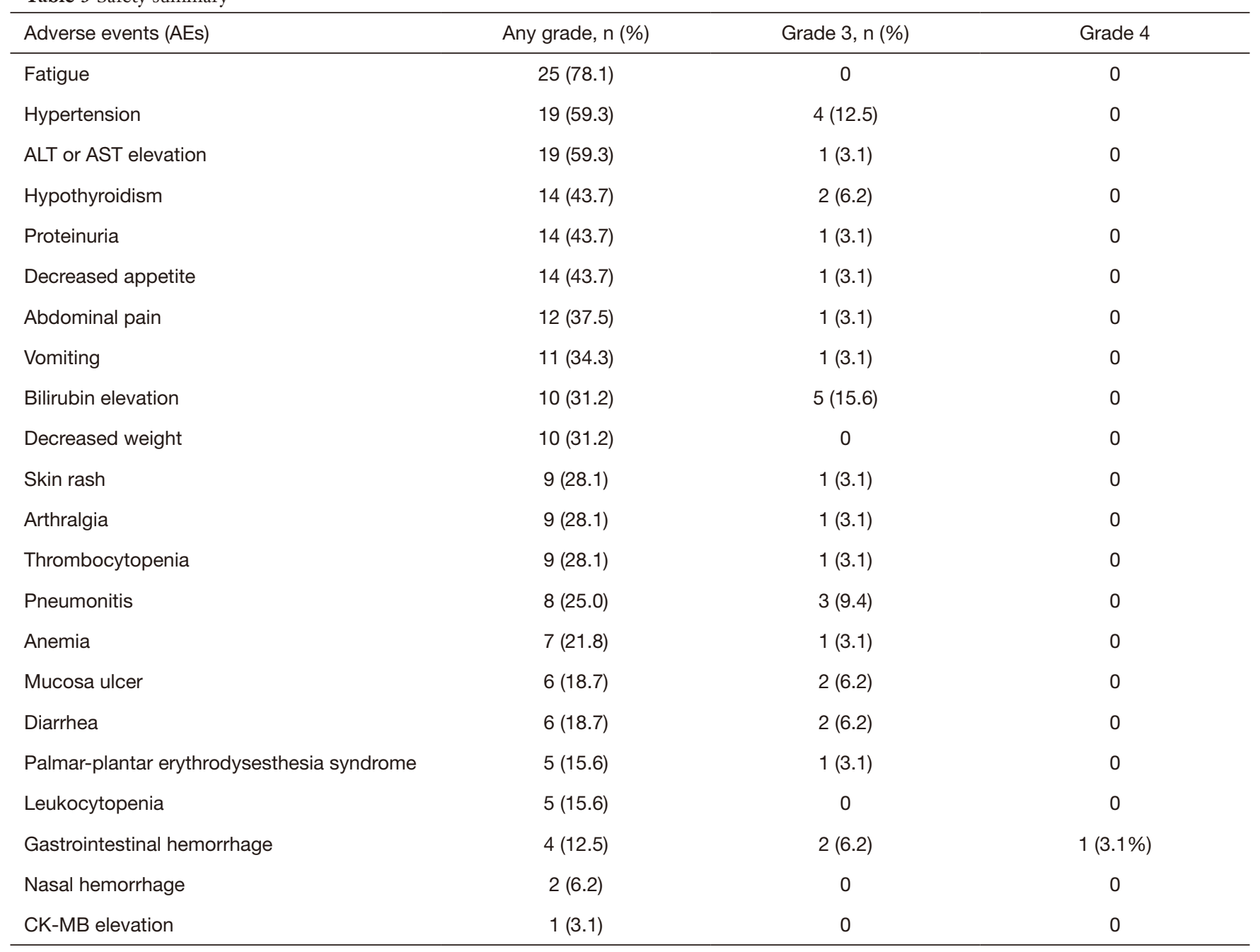

ALT, alanine aminotransferase; AST, aspartate aminotransferase; CK-MB, creatine kinase myocardial band.

(78.1\%), hypertension (59.3\%), elevated aminotransferase (ALT or AST) levels (59.3\%), proteinuria (43.7\%), hypothyroidism (43.7\%) and decreased appetite (43.7\%). Most AEs occurring during combination immunotherapy were safe, well tolerated and controlled. Regarding SAEs, the most common grade 3 SAEs were elevated bilirubin levels $(15.6 \%)$, followed by hypertension $(12.5 \%)$ and pneumonitis $(9.4 \%)$. The elevated bilirubin levels were suspected to be caused by disease progression with lesions occupying bile duct.

\section{Assessments of PDL1 expression and subgroup analyses}

Post hoc subgroup analyses of clinicopathological factors are presented in the forest plot (Figure 2A). As one of the leading efficacy-related biomarkers, PDL1 expression was also retrospectively assessed in available pre-LEP treatment tumor tissues of all 32 patients (Figure 2B). Among these patients, $34.4 \%(11 / 32)$ were determined to have positive PDL1 expression. The subgroup with positive PDL1 expression showed a non-significantly higher ORR than the subgroup with negative PDL1 expression (36.4\%, 4/11 vs. 19\%, 4/21, P=0.397). Patients with positive PDL1 expression had a significantly higher CBR than patients with negative PDL1 expression $(72.7 \%$, 8/11 vs. 23.8\%, $5 / 21, \mathrm{P}=0.021)$. Consequently, patients with positive PDL1 expression showed significantly improved survival outcomes in both PFS and OS, suggesting that PDL1 expression was a potential prognostic factor. When patients were stratified by PDL1 expression, Kaplan-Meier survival curve 


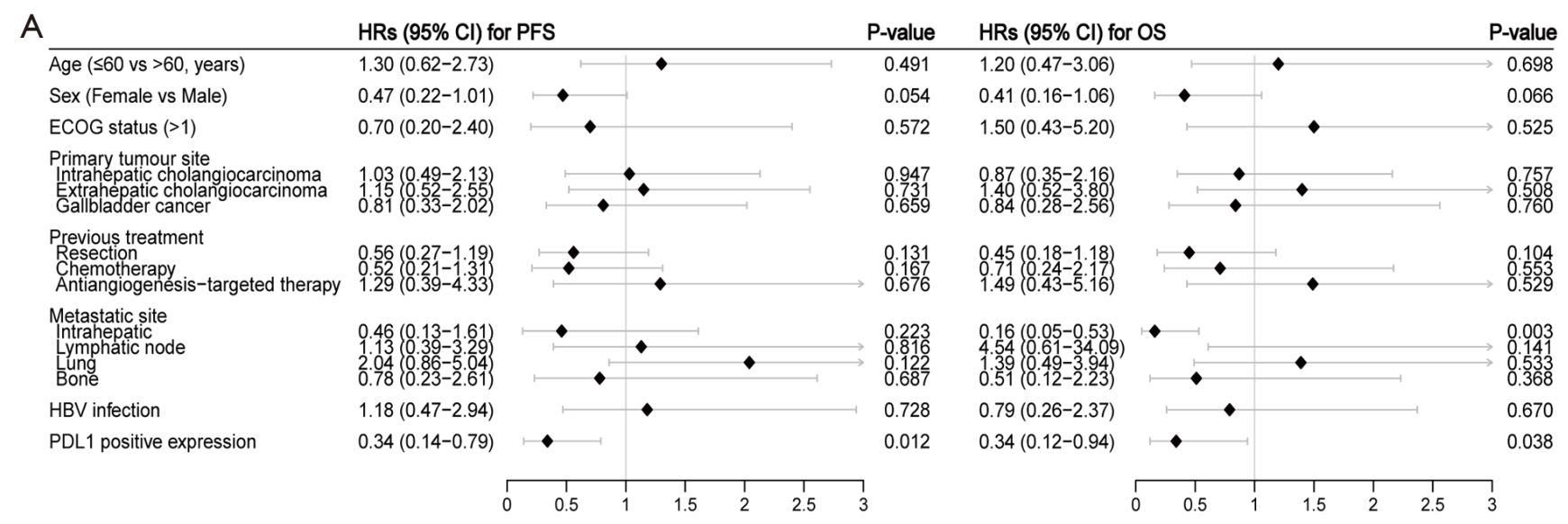

B
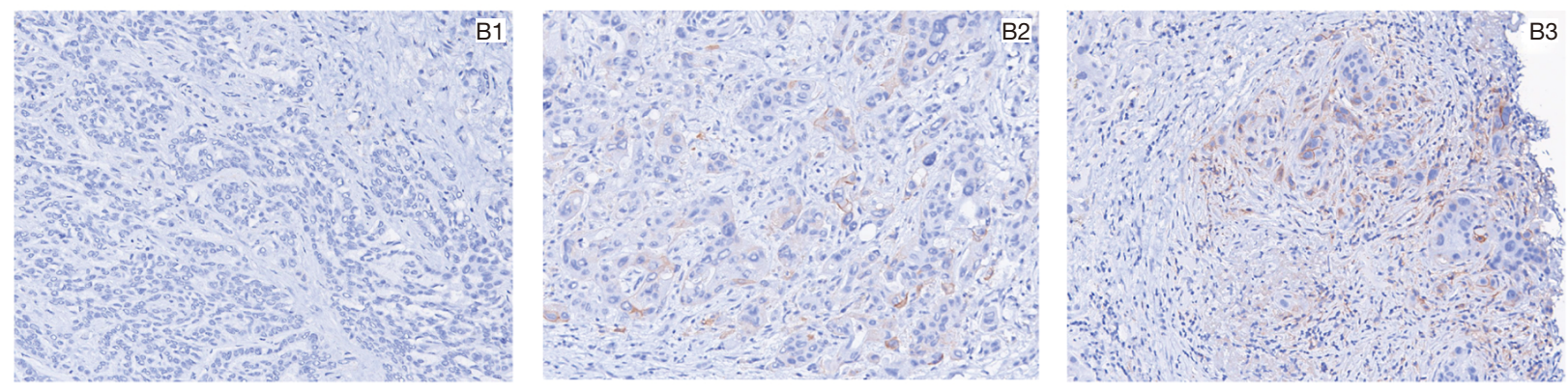

C

Progression-free survival (PFS, months)

D

Overall survival (OS, months)
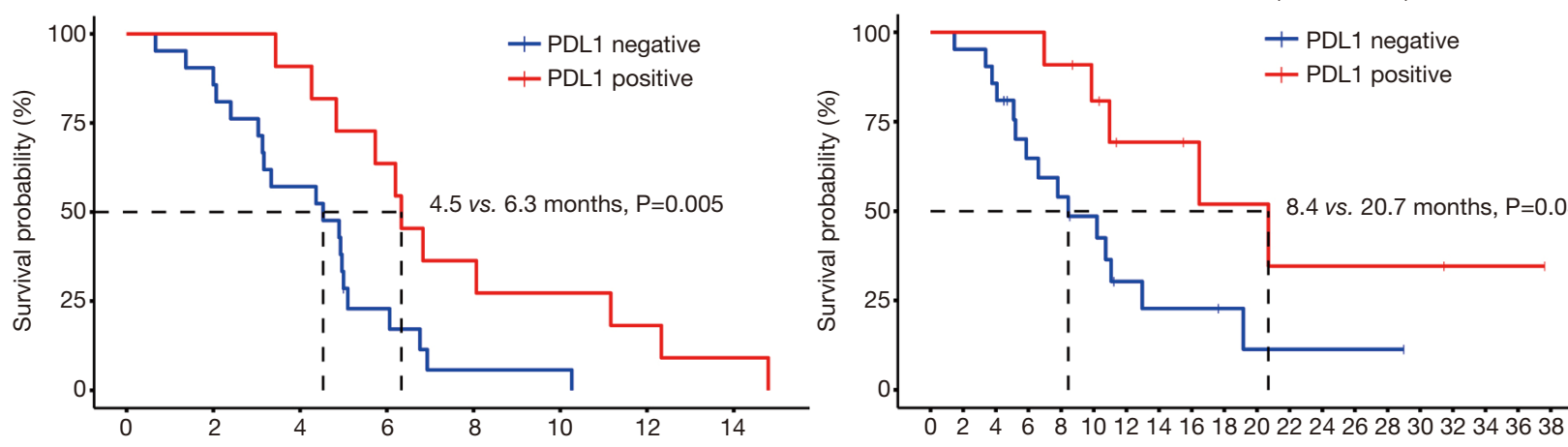

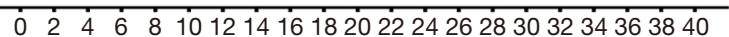

\section{Patients' number at risk}

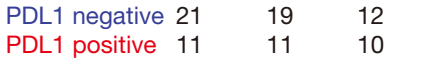

14

\section{Patients' number at risk}

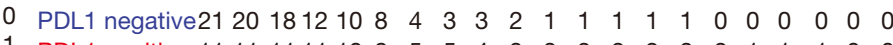

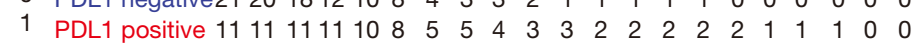

Figure 2 Association of the PDL1 expression detected by IHC staining with survival outcomes. (A) Subgroup analyses of progressionfree survival (PFS) and overall survival (OS) in the entire population; (B) representative photomicrographs (40× objective) of PDL1 immunohistochemistry in patents' archived pretreatment formalin-fixed and paraffin-embedded tumor tissue: negative PDL1 expression (no PDL1-stained tumor cell, B1; PDL1-stained tumor cell <5\%, B2), positive PDL1 expression (PDL1-stained tumor cell >5\%, B3); (C) among the 32 patients with evaluable PD-L1 expression and available radiological assessments, the group with positive PDL1 expression $(n=11)$ showed a prolonged progression-free survival compared with the group with negative PDL1 expression ( $\mathrm{n}=21$ ); (D) a significantly longer overall survival was observed in the group with positive PD-L1 expression. ECOG, Eastern Cooperative Oncology Group; HBV, hepatitis B virus; HR, hazard ratio. 
Table 4 Comparisons of major clinicopathological features between patients with different responsive status and PDL1 expression

\begin{tabular}{|c|c|c|c|c|c|c|}
\hline Parameters & Responder & Non-responder & $P$ value & PDL1 positive & PDL1 negative & $P$ value \\
\hline Gender, n (\%) & & & 0.412 & & & 0.266 \\
\hline Female & $2(25.0)$ & $12(50.0)$ & & $3(27.3)$ & $11(52.3)$ & \\
\hline Male & $6(75.0)$ & $12(50.0)$ & & $8(72.7)$ & $10(47.7)$ & \\
\hline $0-1$ & $7(87.5)$ & $21(87.5)$ & & $10(91.0)$ & $18(85.7)$ & \\
\hline 2 & $1(12.5)$ & $3(12.5)$ & & $1(9.0)$ & $3(14.3)$ & \\
\hline Primary tumor site, $\mathrm{n}(\%)$ & & & 0.206 & & & 0.439 \\
\hline ICC & $4(50.0)$ & $12(50.0)$ & & $6(54.5)$ & $10(47.7)$ & \\
\hline \multicolumn{7}{|l|}{ Previous antitumor therapy, $\mathrm{n}(\%)$} \\
\hline Radical surgery resection & $7(87.5)$ & $10(41.7)$ & 0.041 & $7(63.6)$ & $10(47.7)$ & 0.472 \\
\hline Systemic chemotherapy & $7(87.5)$ & $19(79.2)$ & 1 & $11(100.0)$ & $15(71.4)$ & 0.071 \\
\hline Target-angiogenic therapy & $1(12.5)$ & $2(8.3)$ & 1 & 0 & $3(14.3)$ & 0.534 \\
\hline \multicolumn{7}{|l|}{ Metastatic site, n (\%) } \\
\hline Intrahepatic & $8(100.0)$ & 20 (83.3) & 0.55 & $10(91.0)$ & $18(85.7)$ & 1 \\
\hline Lymph nodes & $6(75.0)$ & $22(91.7)$ & 0.254 & $9(82.0)$ & 19 (90.5) & 0.593 \\
\hline Lung & $1(12.5)$ & $6(25.0)$ & 0.646 & $2(18.2)$ & $5(23.8)$ & 1 \\
\hline
\end{tabular}

and log-rank test analysis demonstrated that patients with positive PDL1 expression had a longer median PFS (6.3 vs. 4.5 months, $\mathrm{P}=0.005$, Figure $2 C$ ) and a prolonged median OS (20.7 vs. 8.4 months, $\mathrm{P}=0.03$, Figure $2 D)$. Furthermore, we compared the clinicopathological characteristics between patients grouped by objective response (responders/ non-responders) or PDL1 expression (PDL1 positive/ PDL1 negative). We noticed that a greater proportion of responders had a previous resection history than nonresponders (Table 4), suggesting that patients with resected BTC at the time of initial diagnosis may have disease that is more responsive to LEP.

\section{Discussion}

Compared with the published literature, this is the first study to analyze therapeutic outcomes in patients receiving treatment with pembrolizumab combined with lenvatinib as a non-first-line therapy for advanced BTC. In the present study, we describe the antitumor activity and toxicity of lenvatinib plus pembrolizumab as a non-first-line treatment in 32 patients with advanced BTC. Most patients enrolled in our study cohort were at a late clinical stage and were estimated to have a limited survival time. All patients were pretreated with systemic antitumor treatments, among whom $47 \%$ (15/32) received three or more previous therapies. Nevertheless, these patients achieved an ORR of $25 \%$ and a CBR of $40.5 \%$, with a median PFS of 4.9 (95\% CI: 4.7-5.2) months and a median OS of 11.0 (95\% CI: 9.6-12.3) months. In terms of therapeutic safety, although all patients experienced AEs, there were no grade 5 SAEs reported, and only 1 patient experienced a grade 4 SAE. Approximately $59.3 \%$ of patients experienced grade 3 SAEs, but all these SAEs were reversible, which is similar 
with the outcomes found in hepatocellular carcinoma (18). Our preliminary study reveals that pembrolizumab combined with lenvatinib is potentially effective and tolerable as a systemic therapy for patients with refractory BTC, highlighting the need to develop a prospective and randomized-control clinical trial to evaluate this regimen in advanced BTC.

Accumulating preclinical studies and clinical evidence demonstrate that inhibition of angiogenesis by targeting VEGF/VEGFR can enhance the antitumor efficacy of immunotherapy targeting PD1/PDL1 (19,20). Lenvatinib, an oral agent, is a multikinase inhibitor targeting VEGFR 1 to 3, FGFR 1 to 4, platelet-derived growth factor receptor- $\alpha$, and RET and KIT proto-oncogenes (21). A phase 2 trial (NCT02579616) showed that lenvatinib as a second-line treatment in patients with unresectable BTC could achieve a $12 \%$ ORR and a $46 \%$ DCR (22). Pembrolizumab used in patients with advanced BTC had an ORR of $13-17 \%$ (KEYNOTE-028) $(15,16)$. Thus, such as most drugs used as second-line treatment, the efficacies of these two agents are not satisfactory when they are used as single agent therapy in patients with refractory BTC. So far, non-first-line therapies have poor track records for BTC. Our data preliminarily offers an alternative strategy of combination immunotherapy for patents with chemotherapy-resistant or uncontrollable BTC.

Due to the complexity and heterogeneity of the tumor immune microenvironment, predictive biomarkers for combination immunotherapy are ambiguous. The role of PD-L1 expression as a combination immunotherapy biomarker remains controversial (23). In our study, by analyzing efficacy-related factors, we found that positive PDL1 positive expression in pretreated tumor tissues was significantly associated with a higher CBR and improved PFS and OS. Considering the limited sample size, the present correlation between PDL1 expression status and therapeutic outcomes should be cautiously interpreted. Underlying confounding factors, such as baseline metastatic disease, tumor mutation burden (TMB) and glucocorticoid use during treatment to relieve immune-related AEs, could affect the predictive effect of PDL1. Thus, predictive biomarkers for combined immunotherapy with LEP require further research in BTC.

The limitations of our exploratory analysis must be acknowledged, highlighting the need for well-designed clinical trials with control arms to determine the precise efficacy and safety of this combined regimen in a prospective interventional setting. Our present study is an investigator-initiated study with underlying selection bias and participant bias, and there was no randomized assignment for the dosages of pembrolizumab $(3 \mathrm{mg} / \mathrm{kg}$ body weight or $200 \mathrm{mg}$ every 3 weeks). Considering the high proportion of PDL1 overexpression $34.4 \%$ of all patients), it is possible that the encouraging efficacy in our cohort partly originated from the selected population, since patients with high PDL1 expression were more likely to be offered with an anti-PD1 inhibitor. Furthermore, AEs, in particular grade 3 AEs, were frequently reported in our cohort, suggesting that the presence of underlying liver disease and the physical condition of the patients with metastatic BTC must be considered prior to initiating this therapeutic regimen. Thus, we believe that LEP is still an immature treatment that should not be considered an alternative option to replace first-line systemic chemotherapy or routinely clinically used in patients with advanced BTC.

In conclusion, we preliminarily reported combined treatment with lenvatinib plus pembrolizumab in patients with refractory BTC. PDL1 overexpression in over $5 \%$ of PDL1-stained tumor cells is an underlying factor that favored improved efficacy outcomes. Further investigation of this therapeutic regimen in prospective clinical

\section{Acknowledgments}

We thank the patients who volunteered to participate in this study and the staff members at the study site who cared for these patients; the members of the data and safety monitoring committee; and the representatives of the sponsors who were involved in the data collection and analyses. We sincerely thank Professor Milind Javle for his kind help guiding the trial design and modifying the manuscript.

Funding: This work was supported by grants from the International Science and Technology Cooperation Projects (2016YFE0107100 and 2015DFA30650), CAMS Innovation Fund for Medical Science (CIFMS) (2017-I2M-4-003), Beijing Natural Science Foundation (L172055), National Ten-thousand Talent Program, Beijing Science and Technology Cooperation Special Award Subsidy Project and CAMS Initiative for Innovative Medicine (CAMS2018-I2M-3-001). The funding source had no role in the design and conduct of the study; collection, management, analysis, and interpretation of the data; preparation, review, or approval of the manuscript; and decision to submit the manuscript for publication. 


\section{Footnote}

Reporting Checklist: The authors have completed the CONSORT reporting checklist. Available at http://dx.doi. org/10.21037/hbsn-20-338

Data Sharing Statement: Available at http://dx.doi. org/10.21037/hbsn-20-338

Conflicts of Interest: All authors have completed the ICMJE uniform disclosure form (available at http://dx.doi. org/10.21037/hbsn-20-338). Drs. YM, XS, and HZ serve as unpaid editorial board members of Hepatobiliary Surgery and Nutrition. SZ, WS and KW report personal fees of OrigiMed Co., Ltd, due to employments outside the submitted work. KW is a grant holder to this company. The other authors have no conflicts of interest to declare.

Ethical Statement: The authors are accountable for all aspects of the work in ensuring that questions related to the accuracy or integrity of any part of the work are appropriately investigated and resolved. The protocol of this study was compliant with the principles of the Declaration of Helsinki and was also approved by the Institutional Review Board (IRB) and Ethics Committee (EC) of Peking Union Medical College Hospital (PUMCH). All participants were asked to provide written informed consent.

Open Access Statement: This is an Open Access article distributed in accordance with the Creative Commons Attribution-NonCommercial-NoDerivs 4.0 International License (CC BY-NC-ND 4.0), which permits the noncommercial replication and distribution of the article with the strict proviso that no changes or edits are made and the original work is properly cited (including links to both the formal publication through the relevant DOI and the license). See: https://creativecommons.org/licenses/by-nc-nd/4.0/.

\section{References}

1. Patel T. Increasing incidence and mortality of primary intrahepatic cholangiocarcinoma in the United States. Hepatology 2001;33:1353-7.

2. Bertuccio P, Malvezzi M, Carioli G, et al. Global trends in mortality from intrahepatic and extrahepatic cholangiocarcinoma. J Hepatol 2019;71:104-14.

3. Khan SA, Toledano MB, Taylor-Robinson SD.
Epidemiology, risk factors, and pathogenesis of cholangiocarcinoma. HPB (Oxford) 2008;10:77-82.

4. Witjes CD, Karim-Kos HE, Visser O, et al. Intrahepatic cholangiocarcinoma in a low endemic area: rising incidence and improved survival. HPB (Oxford) 2012;14:777-81.

5. Anderson C, Kim R. Adjuvant therapy for resected extrahepatic cholangiocarcinoma: a review of the literature and future directions. Cancer Treat Rev 2009;35:322-7.

6. Marino D, Colombi F, Ribero D, et al. Targeted agents: how can we improve the outcome in biliary tract cancer? Hepatobiliary Surg Nutr 2013;2:31-3.

7. Valle J, Wasan H, Palmer DH, et al. Cisplatin plus gemcitabine versus gemcitabine for biliary tract cancer. $\mathrm{N}$ Engl J Med 2010;362:1273-81.

8. Rizvi S, Khan SA, Hallemeier CL, et al. Cholangiocarcinoma - evolving concepts and therapeutic strategies. Nat Rev Clin Oncol 2018;15:95-111.

9. Saeed A, Park R, Al-Jumayli M, et al. Biologics, Immunotherapy, and Future Directions in the Treatment of Advanced Cholangiocarcinoma. Clin Colorectal Cancer 2019;18:81-90.

10. Zhang B, Song Y, Fu Y, et al. Current status of the clinical use of PD-1/PD-L1 inhibitors: a questionnaire survey of oncologists in China. BMC Cancer 2020;20:86.

11. Chen WX, Li GX, Hu ZN, et al. Significant response to anti-PD-1 based immunotherapy plus lenvatinib for recurrent intrahepatic cholangiocarcinoma with bone metastasis: A case report and literature review. Medicine 2019;98:e17832.

12. Smith-Cohn MA, Gill D, Voorhies BN, et al. Case report: pembrolizumab-induced Type 1 diabetes in a patient with metastatic cholangiocarcinoma. Immunotherapy 2017;9:797-804.

13. Hao Z, Wang P. Lenvatinib in Management of Solid Tumors. Oncologist 2020;25:e302-10.

14. Vienot A, Neuzillet C. Cholangiocarcinoma: the quest for a second-line systemic treatment. Transl Cancer Res 2019;8:S275-88.

15. Bang YJ, Doi T, Braud FD, et al. 525 Safety and efficacy of pembrolizumab (MK-3475) in patients (pts) with advanced biliary tract cancer: Interim results of KEYNOTE-028. Eur J Cancer 2015;51:S112.

16. Bang YJ, Ueno M, Malka D, et al. Pembrolizumab (pembro) for advanced biliary adenocarcinoma: Results from the KEYNOTE-028 (KN028) and KEYNOTE-158 (KN158) basket studies. J Clin Oncol 2019;37:abstr 4079.

17. Schwartz LH, Seymour L, Litiere S, et al. RECIST 
1.1 - Standardisation and disease-specific adaptations: Perspectives from the RECIST Working Group. Eur J Cancer 2016;62:138-45.

18. Llovet J, Shepard KV, Finn RS, et al. 747PA phase Ib trial of lenvatinib (LEN) plus pembrolizumab (PEMBRO) in unresectable hepatocellular carcinoma (uHCC): Updated results. Ann Oncol 2019. doi: 10.1093/annonc/ mdz247.073.

19. Shigeta K, Datta M, Hato T, et al. Dual PD-1 and VEGFR-2 blockade promotes vascular normalization and enhances anti-tumor immune responses in HCC. Hepatology 2020;71:1247-61

20. Galon J, Bruni D. Approaches to treat immune hot, altered and cold tumours with combination immunotherapies. Nat Rev Drug Discov 2019;18:197-218.

21. Schlumberger $M$, Tahara $M$, Wirth LJ, et al. Lenvatinib versus placebo in radioiodine-refractory thyroid cancer. $\mathrm{N}$ Engl J Med 2015;372:621-30.

22. Ikeda M, Sasaki T, Morizane C, et al. 722PA phase 2 study of lenvatinib monotherapy as second-line treatment in unresectable biliary tract cancer: Primary analysis results. Ann Oncol 2017. doi: 10.1093/annonc/mdx369.106.

23. Zou W, Wolchok JD, Chen L. PD-L1 (B7-H1) and PD-1 pathway blockade for cancer therapy: Mechanisms, response biomarkers, and combinations. Sci Transl Med 2016;8:328rv4.

Cite this article as: Lin J, Yang $\mathrm{X}$, Long J, Zhao S, Mao J, Wang D, Bai Y, Bian J, Zhang L, Yang X, Wang A, Xie F, Shi W, Yang H, Pan J, Hu K, Guan M, Zhao L, Huo L, Mao Y, Sang X, Wang K, Zhao H. Pembrolizumab combined with lenvatinib as non-first-line therapy in patients with refractory biliary tract carcinoma. HepatoBiliary Surg Nutr 2020;9(4):414424. doi: $10.21037 / \mathrm{hbsn}-20-338$ 